\title{
Optical transfer in space communication
}

\author{
Andrea Farkasvölgyi and István Frigyes
}

\begin{abstract}
This paper presents the possibilities of Free-space optical connection (FSO) in space communication in case of satellite-to-spacecraft or satellite-to-satellite link. It summarizes the advantages and disadvantages of optical transmission in case of near-earth and deep space region. We present the most significant problems during FSO link application and introduce some techniques to eliminate the drawbacks. In this paper, we introduce the use of multi-channel FSO system, which is the most appropriate in order to maximize channel parameters with minimizing the transmission error. It compares available maximum channel parameters of near future space missions. Under special conditions in satellite-earth connection, the usage of the optical link may become very uncertain, because of the strong turbulent medium. We describe conditions under which the optical link can be applied in satellite communication and when it is necessary to effectively modify the optical connection. This article discusses the application of multichannel or more precise diversity systems, which we recommend for space communication.
\end{abstract}

Index Terms-FSO, satellite communication, atmosphere effect, space diversity, non-direct satellite links

\section{INTRODUCTION}

In space communications, the optical transmission has been applied since the mid-90s (SPOT-4 OPALE, OICET). This first inter-satellite optical communication link was a short distance, non-continuous operational transfer link between ARTEMIS GEO (Geostationary orbit) telecommunications satellite and SPOT-4 LEO (Low Earth orbit) Earth observation satellite. This inter satellite connection was a really high capacity data transfer link between two Earth's orbit (LEOGEO 50Mbps, GEO-Ground 780Mbps). The structure of the system is shown in Fig.1.

The major challenge for an Earth observation satellite system is to transfer the collected data by observation equipment to a ground terminal. Due to the huge amount of data and since the satellite appears for about 10 minutes in each rotation period GEO repeater satellites are used [1] [2].

The FSO connections currently used in space communication are near-earth, near space, and deep space communication links. Near-earth communication links are ground-to-satellite or satellite-to-ground links, inter-satellite links, and interorbital links. So these connections are very short distance links $(\max \sim 80.000 \mathrm{~km}$ ) in contrast to the near space or deep space communication links.

The authors are with Department of Broadband Infocommunication and Electromagnetic Theory, Budapest University of Technology and Economics, Hungary; (e-mail:andrea.farkasvolgyi@hvt.bme.hu, frigyes@mht.bme.hu)
The International Telecommunication Union (ITU) defines deep space as a distance that is larger than 2 million $\mathrm{km}$, so Moon-Earth distance and Lagrangian points L1 and L2 are still in near space. Comparing the near-earth and deep space communication, the extra path loss is minimum $60-80 \mathrm{~dB}$, this proportional to the square of the link distance [3].

In deep space communication, the goal is to maximize the transmission data rate, even if the frequency bandwidth is limited, because of the huge background noise. Due to the characteristics of long-distance or deep space communication channel, relative large transmission power, high receiver sensitivity, virtually error-free transmission and high-security channel are essentially required. In the case of near-earth and near space communication, the channel requirements are high data rate, consequently high bandwidth, and also high security [4] [5].

The article is structured as follows: We summarize in Chapter II the advantages and disadvantages of optical transmission. Chapter III includes the negative impacts to be addressed in inter-satellite and inter-spacecraft connections and the elimination and reduction techniques of these effects. In the second part of this chapter, we summarize the possible channel parameters for long-distance space optical links. Chapter IV is a brief analysis of mitigation techniques, possible coding, modulation and diversification options. The final chapter summarizes the possibility of minimizing the negative impact of the atmosphere.

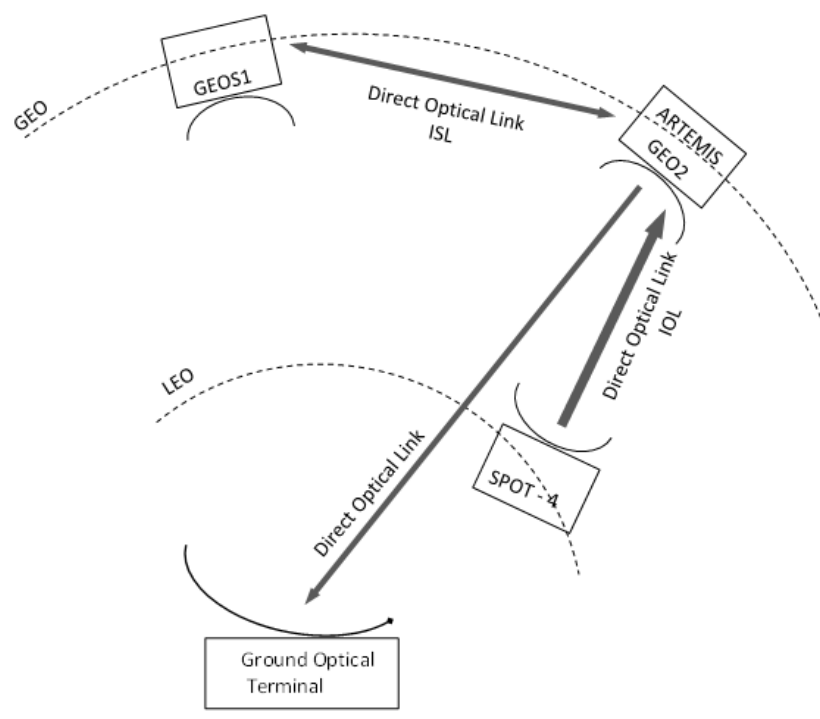

Fig.1 Inter Satellite Communication System; SPOT4 and ARTEMIS 


\section{ADVANTAGES AND DISADVANTAGES OF FREE SPACE OPTICAL COMMUNICATION}

Compared the optical with the radio frequency (RF) communication, the differences come from dissimilar wavelengths. The regular wavelength for RF communication is between $30 \mathrm{~mm}$ to $3 \mathrm{~m}$, compared to the optical $500 \mathrm{~nm}$ $1600 \mathrm{~nm}$. Therefore, RF wavelength is thousands of times larger than the optical wavelength. Differences due to diverse wavelengths are as follows.

\section{A. Advantages of optical communication}

Achievable high data rate: In RF and microwave communication systems, the allowable bandwidth is maximum $0.1 \%-5 \%$ of the carrier frequency, based on [27]. In optical communication, if the bandwidth is $1 \%$ of the carrier frequency, the allowable bandwidth will be $2 \mathrm{THz}$. This makes the usable bandwidth at an optical frequency in the order of $\mathrm{THz}$ which is almost 50000 times that of a typical RF bandwidth. This results in high data rate due to high bandwidth and high effective isotropic radiated power (EIRP) because of the small divergence of the optical beam [3][4].

Lightweight and small size devices: In general, optical devices are much smaller and lighter than RF units. There is a big difference in the size of the radiation units (antennas and optical lens). The beam divergence is proportional to $\lambda / D_{R}$, where $\lambda$ is the carrier wavelength and $D_{R}$ the aperture diameter of the antenna or optical lens. The beam spread offered by the optical carrier is narrower than that of the RF carrier. This leads to an increase in the intensity of the signal at the receiver for a given transmitted power. Since the optical wavelength is very small, a very high directivity and large antenna gain can be achieved [6].

Free spectrum utilization: The optical system is free from spectrum licensing till now. This reduces set up and development cost [5][28].

High Security: In FSO communication no detection is possible without a professional instrument as result high directional laser beam with very narrow beam divergence [7]. In addition, the professional tool should be placed in the optical link to the illegal reception.

\section{B. Disadvantages of optical communication}

Required tight ATP system: The most fundamental disadvantage is the requirement of very tight acquisition, tracking and pointing (ATP) system due to the narrow beam divergence [5].

Effect of turbulence: FSO communication is dependent on varying atmospheric conditions that can degrade fatally the system performance. Therefore, the negative impact of turbulence must be considered near the ground, e.g. attenuation of fog, cloud, rain, dust etc [6].

Sun position: Another limiting factor is the position of the Sun relative to the laser transmitter and receiver. Solar background radiations can increase and that will lead to poor system efficiency [7].

Bandwidth limitation: In the case of long-distance communication it is necessary to apply bandwidth limited signal, it is about $0.1 \mathrm{~nm}(1 \AA)$. Thus, the level of received noise can be reduced to a tolerable level [8].

\section{CHANNEL CONDITION}

The negative effects that influence the signal propagation are different in the turbulent atmosphere and in the free space environment. Thus, it is necessary to examine the two different environments: ground station-satellite and satellitesatellite connection.

\section{A. Ground-to-space link}

Various factors cause absorption and scattering in FSO system, but the major contribution for atmospheric attenuation is due to fog, clouds and water vapor. Eliminate the negative effect of different weather conditions that may perhaps cause huge losses is a fundamental objective. During dense fog conditions, when the visibility is even less than $50 \mathrm{~m}$, attenuation can be more than $350 \mathrm{~dB} / \mathrm{km}$. Fog can extend vertically up to the height of $400 \mathrm{~m}$ above the Earth's surface, so the maximum attenuation due to for is $140 \mathrm{~dB}$. Similar losses and other problems are caused by the cloud cover. Therefore the ground terminal is installed in a location where the probability of the problematic weather conditions (fog, cloud, water vapor) are small and its degree can be determined (based on reliable long-term measurement data) [6].

We should mention the powerful effects of the atmospheric turbulence. The channel parameters depend on the condition of the atmosphere. Basically, turbulence intensity can affect the probability of fade. If the receiving unit is provided with tight ATP system and the beam is collimated then the transmission channel parameter will be appropriate.

Beam wandering does not occur for downlink channels. However, for uplink at the satellite, it can be as large as several microradians. Beam wander can be mitigated by the use of multiple beams or a fast-tracking transmitter [5].

Pointing errors cause serious degradation in the communication channel reliability e.g. off-axis scintillation and deteriorate the outage probability. For downlink aperture averaging will occur for sufficiently large receiver apertures, but for uplink at the satellite, it does not occur this is why any receiver at the satellite always behaves like a point receiver.

Due to the presence of turbulence in the atmosphere, the laser beam wavefront arriving at the receiver will be distorted this effect is called angle-of-arrival fluctuation. This will lead to spot motion or image dancing at the focal plane of the receiver. For downlink, the rms (root mean square) angle-ofarrival fluctuations are several microradians, and for uplink at the satellite is generally less than $1 \mathrm{mrad}$ [9].

Beam spreading causes a dilution of the available power for an uplink channel. Beam scintillation leads to redistribution of signal energy resulting in temporal and spatial irradiance fluctuations of the received signal. This intensity fluctuation of the received signal is known as scintillation and is the major cause of degradation in the performance of the FSO system.

The background noise has to be mentioned. The main sources of background noise are: diffused extended background noise from the atmosphere, background noise from the Sun and other stellar (point) objects and scattered light collected by the receiver. Typically in long-distance communication the background noise can be controlled by limiting the receiver optical bandwidth. Single optical filter with very narrow bandwidth in the order of approx. $0.01 \mathrm{~nm}$ 
can be used to control the amount of background noise. Some of the design considerations while selecting narrowband optical filter are the angle of arrival of the signal, Doppler shifted line width of the laser and various temporal modes [10] [14].

\section{B. Space-to-space link}

Space FSO links are not subject to atmospheric and weather limitations, however, they are limited by other challenges like PAA (Point Ahead Angle), Doppler shift, acquisition and tracking, background radiations and satellite platform stability.

In that case when the transmitter and the receiver move relative to each other, the relative movement and the speed difference cause the Doppler shift. This problem must be compensated. Doppler shift is relevant in interorbital nearearth links and in deep space range also. For large distances, the Doppler shift rate is 70 to 100 times larger than in nearearth region. This means that in deep space communication the Doppler frequency shift rate is greater than the bandwidth.

PAA is one of the most critical parameters of the satelliteto-satellite or satellite-spacecraft laser communication that arises due to the relative angular speed of the two terminals. Due to the relative movement of the transceivers, the reflected signal is received with an offset relative to the beacon to hit the receiver in a suitable position. For the duration of the transmission $\left(t_{n+1}\right)$ and the replies from the receiver $\left(t_{n-1}\right)$, the angular movement of the receiving side: $\mathrm{PAA}=2 \mathrm{~V} / \mathrm{c}$, where, $\mathrm{V}$ is relative speed of receiver to transmitter and $\mathrm{c}$ is light speed, see Fig.2. This is independent of the distance of communication, it only depends on the relative angular velocities (LEO track is $7 \mathrm{~km} / \mathrm{s}$, compared with $\sim 50 \mathrm{~km} / \mathrm{s}$ for Mars Earth). To do this, a scanning system is required to quickly scan the transmitter beam and receiver FOV (Field of View) [3][4].

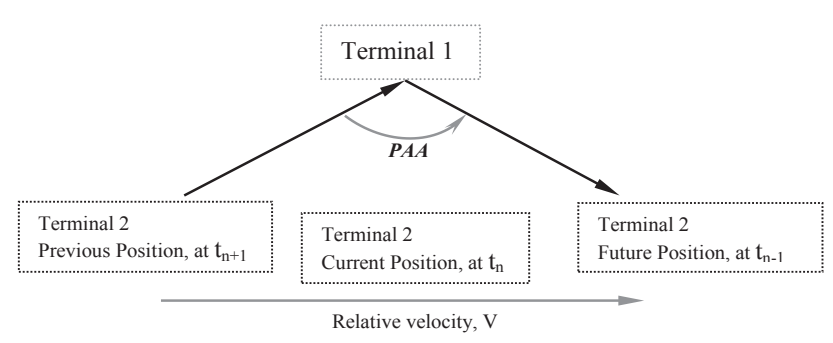

Fig. 2 Most critical parameters in space communication, PAA

The Sun is the source of most background noise in the Solar System. It results in significant scattering if there is a strong background source close to the FOV (Field of View) of the receiver. Degradations caused by radiating celestial bodies other than the Sun are generally negligible except when receiving optics are directly pointing the Sun. The major source of background noise is due to scattering when an optical receiver design has its optics under direct exposure to sunlight [13] [14].

\section{CONNECTION CHARACTERIZATION}

\section{A. Near-earth link}

The near-earth region is close to Earth. It means the Earth's orbits (LEO, MEO, GEO and HEO). These connections are characterized by achievable high data rate that is due to the high allocatable bandwidth. For such small distances, bandwidth limitation is not needed. For this region, the tight ATP system is required. The path loss can be compensated; the link delay is negligible compared to the deep space connections. Both direct and coherent detection links have been demonstrated in space-to-space links and space-toground links. Near-earth links have supported high data rates (> 1Gbps) from space-to-ground and $5.6 \mathrm{Gbps}$ in space-tospace and ground-to-space links. Near-infrared wavelength lasers at discrete wavelengths around 800, 1064 and $1550 \mathrm{~nm}$ have been used.

In the case of space-to-space links, there is no negative effect of the atmosphere and weather conditions. Generally, phase coherent reception techniques are used that deliver high link capacity.

ESA (European Space Agency) has launched a complete and adapted program for support optical communication technologies, called Scylight (SeCure and Laser communication Technology) it is a project in the framework of ARTES, considering possible technologies, systems, and applications. Details about the program can be found on [29] and [30].

Based on recent research [31-33], the use of quantum technology provides tremendous opportunities for near-earth communication. Thus, the Scylight programme concentrates among others quantum cryptography technologies and initial services demonstration.

\section{B. Deep space link}

In space communication, the "long-distance" is usually used for distances of 0.1AU (Astronomical Unit) or more. Usually, the Moon as a celestial is the nearest target, which is already in that distance. In the near future, these links may be implemented in the form of fully optical systems.

In general, it is necessary to use the bandwidth limitation, it is about $1 \AA(0.1 \mathrm{~nm})$ [6], which reduces the background noise to an appropriate level. Tight ATP system is required similar to the shorter near-earth region. Exclusively IM/DD (Intensity Modulation / Direct Detection) is used, where modulations are OOK (On off Keying) for shorter links and PPM (Pulse Position Modulation) for deep space connections. The achievable data rate is strongly dependent on the link distance. For Mars communication, the maximum data rate may reach $0.5 \mathrm{Gbps}$. The communication bands used are identical to the previous case [6] [10].

Let's summarize past and future missions using long-range optical connections. Well-known, full developed NASA mission was the Mars Laser Communications Demonstration (MLCD). It would have been a Mars-Earth high capacity system with RF and optical connections. But this project was suspended before the economic crisis.

NASA's new deep space project will launch in 2021. It is known as Psyche mission, Deep Space Optical 
Communications (DSOC). Psyche is the giant metal asteroid with near circular orbit between Mars and Jupiter.

The goal of this mission is to demonstrate at least a $10 \times$ enhanced data-return capacity relative to state of the art deepspace telecommunication systems with equivalent mass and power; try to achieve $10 \mathrm{Mbps}$ from Saturn and $250 \mathrm{kbps}$ from Neptune by scaling flight transceiver to $0.4 \mathrm{~cm}$ and $20 \mathrm{~W}$; a high-definition video stream transfer from deep-space and use of high data rate science devices [11] [12].

TABLE 1. Realized and future long-range optical space communication systems

\begin{tabular}{|c|c|c|c|c|c|c|}
\hline Mission / instrument & Operation & Target & Link & Data rate (Mbps) & $\begin{array}{l}\text { Distance } \\
(\mathrm{AU})\end{array}$ & $\begin{array}{l}\text { Q - Link difficulty dB } \\
10 \log \left(\mathrm{Mbps} \cdot \mathrm{AU}^{2}\right)\end{array}$ \\
\hline \multirow{2}{*}{$\begin{array}{l}\text { LLCD (Lunar Laser } \\
\text { Com. Demonstration) }\end{array}$} & \multirow{2}{*}{$\begin{array}{l}\text { 10. } 2013- \\
04.2014\end{array}$} & \multirow{2}{*}{$\begin{array}{l}\text { Moon - High band width, } \\
\text { Lunar orbit to Earth } \\
\text { optical link }\end{array}$} & Uplink & 20 & \multirow[b]{2}{*}{$2.7 \mathrm{E}-3$} & -38.36 \\
\hline & & & Downlink & 622 & & -23.43 \\
\hline $\begin{array}{l}\text { OPALS (Optical PAyload } \\
\text { for Lasercomm Science) }\end{array}$ & $04.2014-07.2014$ & $\begin{array}{l}\text { LEO - From IIS to } \\
\text { ground station }\end{array}$ & $\begin{array}{l}\text { Up and } \\
\text { Downlink }\end{array}$ & $20-100$ & $1.33 \mathrm{E}-5$ & $-28.51 \div-77.52$ \\
\hline $\begin{array}{l}\text { LCRD (Laser } \\
\text { Communications Relay } \\
\text { Demonstration) }\end{array}$ & Flight in 2019 & $\begin{array}{l}\text { GEO/Near-earth and } \\
\text { space } \\
\text { High band width } \\
\text { GEO to ground optical } \\
\text { link }\end{array}$ & $\begin{array}{l}\text { Up and } \\
\text { Downlink }\end{array}$ & 1200 & $2.68 \mathrm{E}-4$ & -40.64 \\
\hline \multirow{3}{*}{$\begin{array}{l}\text { DSOC (Deep Space } \\
\text { Optical Communications) }\end{array}$} & \multirow{3}{*}{$\begin{array}{l}\text { Launch Summer of } \\
2022 ; \\
\text { Arrival in } 2026\end{array}$} & \multirow{3}{*}{$\begin{array}{l}\text { Mars/Jupiter - Psyche } \\
\text { mission, distance } 0.1 \\
\text { to2.5AU }\end{array}$} & Downlink & 250 & $2 \mathrm{E}-1$ & 10 \\
\hline & & & Downlink & 250 & $5.5 \mathrm{E}-1$ & 18.8 \\
\hline & & & Uplink & 250 & $2 \mathrm{E}+0$ & 30 \\
\hline
\end{tabular}

Table1. contains the maximum available channel characteristics at different systems and its range in astronomical units $\left(1 \mathrm{AU}=1.49 \cdot 10^{11} \mathrm{~m}\right)[8][15-20]$.

Table 1 introduces an added element of "difficulty" for optical links. Traditionally, the product of data rate and the square of link range (Mbps-AU ${ }^{2}$ ) is used as an index of link difficulty, however, for optical links the proximity to the Sun and the received background noise adds to the difficulty. Relative to near-earth, lasercom from deep space presents link difficulty that increases as the square of the link distance. For example, factors of $60-80 \mathrm{~dB}$ additional gain will be required from Mars distances relative to GEO [8] [10].

\section{REDUCTION OF NEGATIVE EFFECT OF ATMOSPHERE}

As mentioned elsewhere, the basic requirements for space communication channel high data rate and also high security. Turbulence intensity can be reduced channel parameters; the characterization of turbulence is scintillation index. Strong turbulence results in large scintillation index; it is the effective variance of the incident signal [21-25].

Examine the use of multiple channels as a channel parameter correction technique. Using multiple channels system the scintillation can be drastically reduced with several multiple beams for uplink or downlink transmission. If the channels are presumably independent, the rate of decline is inversely proportional to the antenna numbers in the turbulent scenario.

In case of SIMO (single-input and multiple-outputs), these systems utilize the receiver diversity. Diversity gain is achieved by averaging over multiple signal paths. The signals can be combined at the receiver using selection combining (SC) or equal gain combining (EGC) or maximal ratio combining (MRC). Implementation of EGC is preferred over MRC due to its simplicity and suitable channel performance. In case of MISO (transmit diversity) a special space-time code such as optical Alamouti code is used.

As mentioned above, the result of using multiple channels at the ground terminal the scintillation can be reduced. The degree of decline is $1 / \mathrm{M}$ or $1 / \mathrm{N}(\mathrm{M}, \mathrm{N}$ are the antenna number on transceiver of Earth's station). By the improving scintillation index, the channel parameter can also improve. For optical transmission from deep space, multiple receivers can be proposed for secure information transmission, since better channel parameters are available.

Usually, diversity techniques can improve the link performance. In case of the space-diversity the rate of increase in SNR can be determined as follows:

$$
\left\langle\mathrm{SNR}_{\mathrm{M}}\right\rangle=\frac{\mathrm{M}\left\langle\mathrm{I}_{\mathrm{s}, 1}\right\rangle}{\sqrt{\mathrm{M}\left(\sigma_{\mathrm{s}, 1}^{2}+\sigma_{\mathrm{n}, 1}^{2}\right)}}=\sqrt{\mathrm{M}}\left\langle\mathrm{SNR}_{1}\right\rangle
$$

where the number of Earth's transceiver antennas are M and the degree of improvement is $\sqrt{\mathrm{M}}$.

The negative effect of atmospheric turbulence can be reduced by installing receivers on high satellite orbit. Probably it would be a feasible signal reception from deep space by multiple simultaneous satellites receiver antennas. If onboard signal regeneration is available on satellites improved signal is radiated toward the ground terminal. Unfortunately, in the case of satellite-Earth connection, the use of optical links may become very vulnerable under special conditions. The effect of scintillation or attenuation, caused by the atmosphere degrades fatally the efficiency of the channel. That is why in certain cases an additional radio frequency link may be required by which negative effects of turbulence can be minimized. The biggest problem is that the maximum available bit rate of the RF or microwave link is much smaller than the original optical link. It would be a solution to transmit the optical signal by an inter-satellite-link to an orbital position where atmospheric conditions are appropriate for radiation of the optical signal toward the ground terminal. These systems would be a multi-hop communication channel that eliminates the negative effect of atmosphere and ensures the high data rate and high reliability, uninterrupted space link connection. 


\section{CONCLUSION}

This paper presented the properties of free space optical systems which used in space communication. We discussed the preferred properties and drawbacks of FSO in space communication. We presented the most significant problems in optical link application and introduced techniques for drawback eliminations. The paper detailed maximum available channel characteristics especially data rate and Q factor (link difficulty). In our article, the multi-channel application of optical systems was introduced by which maximize the channel parameters and minimize transmission error. Nevertheless, under special conditions, in the satellite to ground station relation, the usage of the optical link may become very uncertain. Usage of RF link reduces the negative impact of turbulence, but this would result in significant bandwidth reduction. An inter-satellite link would be the solution to this problem. Thus, the received signal can be radiated to an orbital position, from where atmospheric conditions are perfect toward a ground terminal. This is a multi-hop optical system that can decrease negative effects of atmosphere and weather conditions and ensures high reliability, uninterrupted space link.

\section{ACKNOWLEDGMENT}

The first author thanks Eva Godor for support and assistance.

\section{REFERENCES}

[1] T. T. Nielsen and G. Oppenhauser, "In-orbit test result of an operational optical intersatellite link between ARTEMIS and SPOT4, SILEX," Proc. SPIE, Free Space Laser Comm. Tech. XIV, vol. 4635, 2002.

[2] A. Katsuyoshi, "Overview of the optical inter-orbit communications engineering test satellite (OICETS) project," J. Nat. Inst. of Info. and Comm. Tech., vol. 59, pp. 5-12, 2012 .

[3] H. Hemmati, A. Biswas, and I. B. Djordjevic, "Deep-space optical communications: Future perspectives and applications," Proc. IEEE, vol. 99, no. 11, pp. 2020-2039, 2011.

[4] H. Hemmati, Deep Space Optical Communication. John Wiley \& Sons, New York, 2006

[5] H. Kaushal, G Kaddoum, "Optical communication in Space: Challenge and Mitigation Techniques". IEEE Communication Surveys and Tutorial, 2017

[6] B. Flecker, M. Gebhart, E. Leitgeb, S. S. Muhammad, and C. Chlestil, "Results of attenuation measurements for optical wireless channels under dense fog conditions regarding different wavelengths," Proc. SPIE, Atmospheric Opt. Model., Measure., and Simula. II, vol. 6303, 2006.

[7] A. Biswas, D.M. Boroson, "Near-Sun free-space optical communications from space," boI: 10.1109/AERO.2006.1655849-Source: IEEE Xplore

[8] Yozo Shoji, et al., "A Pilot-Carrier Coherent LEO-to-Ground Downlink System Using an Optical Injection Phase Lock Loop (OIPLL) Technique," IEEE Journal of Lightwave Technology 2012.

[9] L. C. Andrews and R. L. Phillips, "Laser Beam Propagation through Random Media."SPIE Press, 2005.

[10] H. Kaushal, V. Kumar, A. Dutta, H. Aennam, H. Aennam, V. Jain, S. Kar, and J. Joseph, "Experimental study on beam wander under varying atmospheric turbulence conditions," IEEE Photon. Tech. Lett., vol. 23, no. 22, pp. 1691-1693, 2011.

[11] D.M. Boroson; Chien-Chung Chen; B. Edwards, "Overview of the Mars laser communications demonstration project" LEOS Summer Topical Meetings, 2005, INSPEC A. Num.: 8715041

[12] K.E. Wilson, "An overview of the GOLD experiment between the ETSVI satellite and the table mountain facility," TDA Progress Report 42124, Comm. Sys. and Research Sec.,pp. 9-19, 1996.
[13] G. Baister, K. Kudielka, T. Dreischer, and M. Tüchler "Results from the DOLCE (deep space optical link communications experiment) project," Proc. SPIE, Free Space Laser Comm. Tech. XXI, vol. 7199, 2009.

[14] Hemani, Kaushal, e.a "Ground-to-Satellite Optical Communication Link Performance with Spatial Diversity in Weak Atmospheric Turbulence," Fiber and Integrated Optics, ISSN: 0146-8030 print/1096-4681

[15] D. M. Boroson, A. Biswas, and B. L. Edward, "MLCD: Overview of NASA's Mars laser communications demonstration system," Proc. SPIE, Free Space Laser Comm. Tech. XVI, vol. 5338, 2004.

[16] A. Biswas, D. Boroson, and B. Edwards, "Mars laser communication demonstration: What it would have been," Proc. SPIE, Free Space Laser Comm. Tech. XVIII, vol. 6105, 2006.

[17] https://www.nasa.gov/sites/default/files/atoms/files/fs_dsoc_ factsheet_150 910.pdf, last visit Oct.8 2018.

[18] https://www.nasa.gov/sites/default/files/atoms/files/tglavich_dsoc.pdf, last visit Oct.8 2018

[19] Abhijit Biswas,* Hamid Hemmati, et al.. "Deep-space Optical Terminals (DOT) Systems Engineering," IPN Progress Report 42-183, Nov 15, 2010 - https://ipnpr.jpl.nasa.gov/progress_report/42-183/183A.pdf

[20] Donald Cornwell, "Space-Based Laser Communications Break Threshold," Optics and Photonics News 27(5):24 DoI: $10.1364 / \mathrm{OPN} .27 .5 .000024$

[21] D. M. Boroson, A. Biswas, and B. L. Edward, "MLCD: Overview of NASA's Mars laser communications demonstration system," Proc. SPIE, Free Space Laser Comm. Tech. XVI, vol. 5338, 2004.

[22] A. Biswas, D. Boroson, and B. Edwards, "Mars laser communication demonstration: What it would have been," Proc. SPIE, Free Space Laser Comm. Tech. XVIII, vol. 6105, 2006.

[23] A. J. Hashmi, A. A. Eftekhar, A. Adibi, and F. Amoozegar, "Analysis of adaptive optics-based telescope arrays in a deep-space inter-planetary optical communications link between Earth and Mars," Optics Comm. (Elsevier), vol. 333, pp. 120-128, 2014.

[24] V. Weerackody ; A. R. Hammons ; D. J. Tebben, " Multi-Input MultiOutput Free Space Optical Satellite Communication Links," Information Sciences and Systems, 2007. CISS '07. 41st Annual Conference

[25] W. J. Hurd, B. E. MacNeal, G. G. Ortiz, and R. V. Moe et al., "Exoatmospheric telescopes for deep space optical communications," in Proc. IEEE Aerosp. Conf., (Big Sky, MT), 2006.

[26] R. J. Cesarone, D. Abraham, S. Shambayati, and J. Rush, "Deep-space optical communications visions, trends, and prospects," in Proc IEEE Int. Conf. on Space Opt. Sys., (Santa Monica, CA, U S.), 2011.

[27] Rec. S.1709-1 (01/07), in force

[28] ITU Radio Regulations, Geneva, 15 July 2016.

[29] https://artes.esa.int/scylight/overview, last visit Oct.8 2018

[30] https://artes.esa.int, last visit Oct.8 2018

[31] L. Bacsardi, On the Way to Quantum-Based Satellite Communication, IEEE Communications Magazine 51:(08) pp. 50-55., 2013

[32] Oi Daniel K L et al, CubeSat quantum communications mission, EPJ Quantum Technology, 4: Paper UNSP 6. 20 p. 2017

[33] Tamas Bisztray et al, "The Evolution of Free-Space Quantum Key Distribution” Infocommunications Journal X:(1) pp. 22-30. 2018

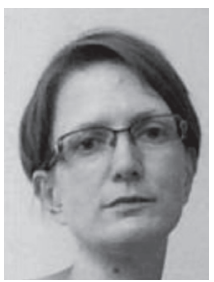

Andrea Farkasvölgyi received her M.Sc. electrical engineer from Budapest University of Technology and Economics. Currently, she is an assistant lecturer at Budapest University of Technology and Economics. Her research focuses on satellite communication, mutual channel techniques.

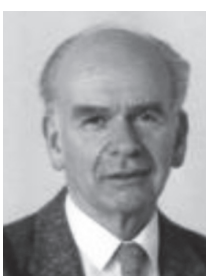

István Frigyes, D.Sc., he is Professor Emeritus at Budapest University of Technology and Economics. His research focuses on satellite communication, propagation effects and system aspects of microwave photonics. He led the Hungarian team of SATNEX and SATNEX-II. He was TPC member of ICCs, GLOBECOMs, he was the general chair of the 2007 IST Mobile Summit of the EU. 